\title{
SUPAC OF IMMEDIATE-RELEASE, MODIFIED- RELEASE AND SEMI-SOLID: A REGULATORY NOTE
}

Available online at www.ijdra.com

\section{REVIEW ARTICLE}

\author{
${ }^{1}$ Ashara Kalpesh C*, ${ }^{2}$ Mendapara Vishal P, ${ }^{2,3}$ Mori Nitin M, ${ }^{4}$ Badjatya J.K. \\ ${ }^{1}$ Department of pharmaceutics, B.K.Mody Govt.Pharmacy College Rajkot, GTU, Gujarat, India. \\ ${ }^{2}$ Department of Pharmaceutical sciences, Saurashtra University, Rajkot, Gujarat, India. \\ ${ }^{3}$ Torrel [Hospital Division] a member of Torrent group, Ahmedabad, Gujarat, India. \\ ${ }^{4}$ Montajat Pharmaceutical Company Limited, Dammam, KSA. \\ *Corresponding Author's E-mail: kalpeshshr5@gmail.com
}

\begin{abstract}
In today scenario, as per market demand there is definitely carry out an increment or decrease in production, this is called SUPAC. Different guidelines are provided for those different types of SUPAC in by different regulatory authority for manufacturing of product. Here SUPAC guidelines for USFDA are elaborated for production in this review article.
\end{abstract}

Keywords: SUPAC-Guideline for IR, MR, SS, SUPAC Regulations..

\section{INTRODUCTION}

- Technology transfer of a pharmaceutical product from research to the production floor with simultaneous increase in production outputs is commonly known as scale - up.

- In simple terms, the process of increasing batch size is termed as scale- up.

- Conversely, scale- down refers to decrease in batch size in response to reduced market requirements.

- Definition: The scale-up process and the changes made after approval in the composition, manufacturing process, manufacturing equipment, and change of site have become known as Scale-Up and Post approval Changes, or SUPAC.

- Changes are being made in the manufacturing process and chemistry of a drug product following approval and continue throughout its life.

- Depending upon foreseen (or unforeseen) requirements, there can be changes in the raw materials, process, equipment or manufacturing site, and batch size which ultimately affect quality attributes of a drug or finished product.
- Therefore, there is a need to anticipate and fully evaluate the impact of any kind of change on the quality of a drug or finished product.

- The intensity of the adverse effect produced by a particular change depends on the type of dosage form.

- For example, a change in the inactive ingredient beyond a certain range will have more effect on a modified- release (MR) dosage form than it would on an immediate - release (IR) dosage form, where bioavailability is not rate limiting.

- Likewise, a change in the primary packaging of liquid Parenteral may have more pronounced effect on its effectiveness than it would have on a solid dosage form.

\section{Scientific \& Regulatory Rational for SUPAC}

Authorities Regulations like FDA (U.S. Food and Drug Administration), the European Commission, the Agencia Nacional de Vigilancia Sanitaria (ANVISA) (in English the National Health Surveillance Agency - Brazil, and others require the pharmaceutical industries in respective 
countries to follow guidelines on scale- up and post approval changes (SUPAC) to maintain the quality of the pharmaceutical produced.

The SUPAC guidance published by the FDA define various levels of change and for each level of change specifies the:

1) Recommended chemistry, manufacturing, and control tests;

2) In-vitro dissolution testing and/or in-vivo bioequivalence tests; and

3) Documentation that the FDA requires to be filed in the NDA, ANDA, or AADA to support the change.(1)

\section{SUPAC-IR - Implementation}

- Federal Register notice - November 30, 1995

- Industry training - February 15, 1996

- Updated via letter - February 18, 1997

- Working Group revising the guidance

- Applies to immediate release tablets, chewable tablets, capsules, and soft gelatin.

\section{SUPAC-MR - IMPLEMENTATION}

-Industry training - November 24, 1997

-Applies to modified release, solid, oral dosage forms

\section{SUPAC-SS - Implementation}

- Federal register notice - June 13, 1997

- Industry training - May 29, 1997

- Applies to non-sterile semi-solid preparations, e.g., creams, gels, and ointments. (2)

\section{METHOD AND ILLUSTRATIONS}

\section{Components \& Compositions Changes:}

Components \& composition changes includes mainly three levels of changes like I, II, III.

Level I Changes: Level 1 changes are minor changes those that are unlikely to have any makeable effect on quality of formulae and the action.

Examples

- deletion of color or flavor

- Excipients change with total additive effect of up to $5 \%$

-Federal register notice - October 6, 1997

Table 1: Allowable percentages changes in Excipients in SUPAC (3)

\begin{tabular}{|l|c|}
\hline Excipients & $\begin{array}{c}\text { Percentage Excipients (w/w) out of total } \\
\text { target dosage form weight }\end{array}$ \\
\hline Filler & \pm 5 \\
\hline Disintegrate & - \\
\hline Starch & \pm 3 \\
\hline Other & \pm 1 \\
\hline Binder & \pm 0.5 \\
\hline Lubricant & - \\
\hline Calcium or magnesium & \pm 0.25 \\
Stearate & \pm 1 \\
\hline Other & - \\
\hline Glidant & \pm 1 \\
\hline Talc & \pm 1 \\
\hline Other & \pm 1 \\
\hline Film coat & \\
\hline
\end{tabular}


Table 2: Test Documentation for Level I Changes (4)

\begin{tabular}{|c|c|c|c|c|}
\hline \multirow[b]{2}{*}{$\begin{array}{c}\text { Test } \\
\text { Documentation }\end{array}$} & \multirow[b]{2}{*}{$\begin{array}{l}\text { IR (Immediate } \\
\text { release) }\end{array}$} & \multirow[b]{2}{*}{$\begin{array}{c}\text { SS } \\
\text { (Semisolid) }\end{array}$} & \multicolumn{2}{|c|}{ MR } \\
\hline & & & $\begin{array}{l}\text { Non-release } \\
\text { controlling } \\
\text { Excipients } \\
\end{array}$ & $\begin{array}{c}\text { Release } \\
\text { controlling } \\
\text { Excipients } \\
\end{array}$ \\
\hline $\begin{array}{l}\text { Chemistry } \\
\text { documentation }\end{array}$ & \multicolumn{4}{|c|}{ Application or compendia release requirement } \\
\hline Stability testing & $\begin{array}{l}\text { One batch with long } \\
\text { term stability testing } \\
(1 \mathrm{LT})\end{array}$ & \multicolumn{3}{|c|}{$1^{\text {st }}$ batch with Long Term stability study } \\
\hline $\begin{array}{l}\text { Dissolution } \\
\text { Documentation } \\
\end{array}$ & \multirow{2}{*}{\multicolumn{4}{|c|}{ None }} \\
\hline In vivo & & & & \\
\hline $\begin{array}{l}\text { Filling } \\
\text { documentation }\end{array}$ & \multicolumn{4}{|c|}{ Annual report (all information including LT) } \\
\hline
\end{tabular}

Level-II Changes: Those that are moderate changes could have significant impact on formulation quality \& performance. Tests \& filling documentations for level-II changes vary depending on three factors: therapeutic range, solubility, permeability, e.g. Change in technical

Table 3: Test Documentation for Level-2 Changes (5) grade of Excipients, Excipients change with total additive effect of up to $10 \%$.Changes in \% of Excipients are as like table 1 \& test documentation for level 2 changes are as follows:

\begin{tabular}{|l|l|l|l|l|}
\hline \multicolumn{1}{|c|}{$\begin{array}{c}\text { Test } \\
\text { Documentation }\end{array}$} & $\begin{array}{c}\text { IR (Immediate } \\
\text { release) }\end{array}$ & \multicolumn{1}{|c|}{$\begin{array}{c}\text { SS } \\
\text { (Semisolid) }\end{array}$} & $\begin{array}{c}\text { Non-release } \\
\text { controlling } \\
\text { Excipients }\end{array}$ & $\begin{array}{c}\text { Release } \\
\text { controlling } \\
\text { Excipients }\end{array}$ \\
\hline $\begin{array}{l}\text { Chemistry } \\
\text { documentation }\end{array}$ & $\begin{array}{l}\text { Release \& } \\
\text { batch record }\end{array}$ & Release \& executed batch record \\
\hline Stability testing & $\begin{array}{l}\text { One batch with 3 months accelerated stability study and one batch } \\
\text { with long term stability study }\end{array}$ \\
\hline $\begin{array}{l}\text { Dissolution } \\
\text { Documentation }\end{array}$ & $\begin{array}{l}\text { Yes depends on permeability \& solubility \& compare as per } \\
\text { dissolution documentations }\end{array}$ \\
\hline In vivo & None & $\begin{array}{l}\text { Non for non-narrow } \\
\text { therapeutic index (T.I.)AND } \\
\text { single dose for narrow T.I. }\end{array}$ \\
\hline $\begin{array}{l}\text { Filling } \\
\text { documentation }\end{array}$ & $\begin{array}{l}\text { PA(Prior Approval all data including accelerated stability data ) } \\
\text { AR(Annual report long term stability data) }\end{array}$ \\
\hline
\end{tabular}

Dissolution documentation is as per below in Table: 4

Table 4: Dissolution documentation of Level-II Changes (6)

\begin{tabular}{|l|l|l|l|}
\hline Cases & \multicolumn{2}{|c|}{ Parameters } & \multicolumn{4}{|c|}{ Documentation } \\
\hline A & $\begin{array}{l}\text { High Solubility } \\
\& \quad \text { high } \\
\text { permeability }\end{array}$ & $\begin{array}{l}85 \% \text { Dissolution in 900 } \mathrm{ml} \text { and 15min of 0.1 N HCl. If a drug product } \\
\text { fails to meet this criterion, the applicant should perform the tests } \\
\text { described for Case B or C as below. }\end{array}$ \\
\hline B & Low & Multipoint dissolution profile should be performed in the \\
\hline
\end{tabular}




\begin{tabular}{|l|l|l|}
\hline permeability \& \\
high solubility \\
C
\end{tabular} \begin{tabular}{l}
$\begin{array}{l}\text { application/compendia medium at 15, 30, 45, 60 and 120 minutes or } \\
\text { until an asymptote is reached. The dissolution profile of the proposed } \\
\text { and currently used product formulation should be similar. } \\
\text { permeability \& } \\
\text { low solubility }\end{array}$ \\
$\begin{array}{l}\text { Multipoint dissolution profiles should be performed in water, 0.1 N } \\
\text { HCl, and USP buffer media at pH 4.5, 6.5 and 7.5 (five separate } \\
\text { profiles) for the suspected and routine accepted formulations. Adequate } \\
\text { sampling should be performed at 15, 30, 45, 60 and 120 minutes until } \\
\text { either 90\% of drug from the drug product is dissolved or an asymptote } \\
\text { is reached. A surfactant may be used, but only with appropriate } \\
\text { justification. The dissolution profile of the proposed and routine used } \\
\text { formulae should be similar. }\end{array}$ \\
\hline
\end{tabular}

Level III Changes: Those that are major changes could have significant impact on formulation quality \& performance

Tests and filing documentation very depending on the following three factors:

- therapeutic range

- solubility

- permeability

Examples:

$>$ Any quality \& quantity changes in narrow prescription drugs.
The different formulations not meeting dissolution criteria.

$>$ Changes in Excipients range of low solubility, low permeability drugs

$>$ Changes in Excipients range of all other drugs.

$>$ Changes in crystalline form of drug substance if drug formulations in suspensions.

$>$ Changes in release controlling Excipients $>10 \%$

$>$ Addition or deletion of release controlling Excipients(s). (7)

Table 5: Test Documentation for Level 3 Changes (8)

\begin{tabular}{|c|c|c|c|c|}
\hline \multirow[b]{2}{*}{$\begin{array}{c}\text { Test } \\
\text { Documentation }\end{array}$} & \multirow[b]{2}{*}{$\begin{array}{l}\text { IR (Immediate } \\
\text { release) }\end{array}$} & \multirow[b]{2}{*}{$\begin{array}{c}\text { SS } \\
\text { (Semisolid) }\end{array}$} & \multicolumn{2}{|c|}{ MR } \\
\hline & & & $\begin{array}{c}\text { Non-release } \\
\text { controlling } \\
\text { Excipients }\end{array}$ & $\begin{array}{l}\text { Release controlling } \\
\text { Excipients }\end{array}$ \\
\hline $\begin{array}{l}\text { Chemistry } \\
\text { documentation }\end{array}$ & $\begin{array}{l}\text { Release \& Batch } \\
\text { records }\end{array}$ & \multicolumn{3}{|c|}{ Release \& executed batch records } \\
\hline Stability testing & $\begin{array}{l}\text { One batch with } 3 \\
\text { months accelerated } \\
\text { stability study and } \\
\text { one batch with } \\
\text { long term stability } \\
\text { study }\end{array}$ & \multicolumn{3}{|c|}{ Same and first three batches with LT } \\
\hline $\begin{array}{l}\text { Dissolution } \\
\text { Documentation }\end{array}$ & $\begin{array}{lr}\text { Yes depends } & \text { on } \\
\text { permeability } & \& \\
\text { solubility } & \& \\
\text { compare as per } \\
\text { dissolution } & \\
\text { documentations } & \\
\end{array}$ & $\begin{array}{l}\text { Not } \\
\text { required }\end{array}$ & Extended and de & \\
\hline In vivo & \multicolumn{4}{|c|}{ Bioequivalence needed } \\
\hline $\begin{array}{l}\text { Filling } \\
\text { documentation }\end{array}$ & \multicolumn{4}{|c|}{$\begin{array}{l}\text { PA(Prior Approval all data including accelerated stability data ),AR(Annual } \\
\text { report long term stability data) }\end{array}$} \\
\hline
\end{tabular}

Extended Release: In addition to application /compendia release requirements, multipoint dissolution profiles should be obtained in three other media, for example, in water, $0.1 \mathrm{~N} \mathrm{HCl}$ and USP buffer media at $\mathrm{pH} 4.5$ and 6.8 for the changed drug product and the bio-batch or 
marketed batch (unchanged drug product). Adequate sampling should be performed, for example, at 1, 2 and 4 hours and every two hours thereafter until either $80 \%$ of the drug from the drug product is released or and asymptote is reached. A surfactant may be used with appropriate justification.

Delayed Release: In addition to application /compendia release requirements dissolution tests should be performed in $0.1 \mathrm{~N} \mathrm{HCl}$ for 2 hours (acid stage) followed by testing in USP buffer media, in the range of $\mathrm{pH}$ 4.5-7.5 (buffer stage) under standard test conditions and two additional agitation speeds using the application/compendia test apparatus (three additional test conditions. If the application/compendia test apparatus is the rotating basked method (apparatus 1), a 50, 100 and 150 RPM rotation speed may be used. Multipoint dissolution profiles should be obtained in between buffer stage of process. Adequate sampling should be performed, for example, at $15,30,45,60$, and 120 minutes (following the time from which the dosage form is placed in the buffer) until either $80 \%$ if the drug from the drug produce is release or an asymptote is reached. The above dissolution testing should be performed using the changed drug product and the bio-batch or marketed batch (unchanged drug product). (9)

Preservatives Changes: It is done with SUPAC-SS only, when quality \& quantity changes are made $\&$ additional testing should be performed.

Table 6: Test Documentation of Preservatives in SUPAC-SS (10)

\begin{tabular}{|l|l|l|l|}
\hline \multicolumn{1}{|c|}{ Test Documentation } & \multicolumn{1}{|c|}{ Level-I } & \multicolumn{1}{c|}{ Level-II } & \multicolumn{1}{c|}{ Level-III } \\
\hline $\begin{array}{l}\text { SUPAC-SS Quantity of } \\
\text { approved preservatives }\end{array}$ & $\begin{array}{l}\leq 10 \% \\
\text { change }\end{array}$ & $\begin{array}{l}>10 \% \leq 20 \% \\
\text { change }\end{array}$ & $\begin{array}{l}\text { >20\% change (including } \\
\text { deletion) or use of a different } \\
\text { preservative }\end{array}$ \\
\hline Chemistry & $\begin{array}{l}\text { Application/compendia release } \\
\text { requirements Preservative } \\
\text { Effectiveness Test }\end{array}$ & $\begin{array}{l}\text { Release requirements, } \\
\text { Preservative Effectiveness } \\
\text { Test, Analytical method for } \\
\text { ID, Validation studies, } \\
\text { Executed batch records }\end{array}$ \\
\hline & AR & $\begin{array}{l}\text { CBE(Change } \\
\text { Being Effected) }\end{array}$ & $\begin{array}{l}\text { PA(Prior Approval all data } \\
\text { including accelerated stability } \\
\text { data ),AR(Annual report long } \\
\text { term stability data) }\end{array}$ \\
\hline Stability testing & - & $\begin{array}{l}\text { One batch with 3 months } \\
\text { accelerated stability study and } \\
\text { one batch with long term } \\
\text { stability study. }\end{array}$ \\
\hline
\end{tabular}

Manufacturing Site Changes: Manufacturing site changes means changes in location sites only, no scale-up, no manufacturing changes, cGMPs.

Manufacturing Site Level I Changes: Level I changes is defined as changes in manufacturing site "within a single facility where the same equipment, SOPs, environmental conditions and controls, and personnel common to both sites are used."

Table 7: Test Documentations of Manufacturing site Level-I Changes

\begin{tabular}{|l|l|l|l|}
\hline \multicolumn{1}{|c|}{$\begin{array}{c}\text { Test } \\
\text { documentations }\end{array}$} & \multicolumn{1}{|c|}{ IR } & SS & MR \\
\hline $\begin{array}{l}\text { Chemistry } \\
\text { documentations }\end{array}$ & Release & \\
\hline
\end{tabular}




\begin{tabular}{|l|l|l|l|}
\hline Dissolution & Release & None & Release \\
\hline In vivo & None & \\
\hline File & Annual Report & \\
\hline
\end{tabular}

Manufacturing Site Level II Changes: Level 2 changes may be defined as the changes in the manufacturing site "within a contiguous campus, or between facilities in adjacent city

blocks, where same equipment, SOPs, environmental conditions and controls, and personnel common to both sites are used"

Table 8: Test Documentations of Manufacturing site Level-II Changes

\begin{tabular}{|l|l|l|l|}
\hline \multicolumn{1}{|c|}{$\begin{array}{c}\text { Test } \\
\text { documentations }\end{array}$} & \multicolumn{1}{|c|}{ IR } & \multicolumn{1}{c|}{ SS } & \multicolumn{1}{c|}{ MR } \\
\hline $\begin{array}{l}\text { Chemistry } \\
\text { documentation }\end{array}$ & $\begin{array}{l}\text { Release; Notification of } \\
\text { location of new site and } \\
\text { updated batch record }\end{array}$ & $\begin{array}{l}\text { Release; Notification of location of new } \\
\text { site and updated executed batch record }\end{array}$ \\
\hline Stability testing & $\begin{array}{l}\text { One long } \\
\text { term }\end{array}$ & First one term & $\begin{array}{l}\text { One batch of three months accelerated } \\
\text { study \& first batch of long term stability } \\
\text { study }\end{array}$ \\
\hline Dissolution & Release & None & Extended \& Delayed \\
\hline In vivo & None & $\begin{array}{l}\text { Change Being Expected (Accelerated study) \& Annual Report (Long term } \\
\text { stability study) }\end{array}$ \\
\hline File &
\end{tabular}

Manufacturing Site Level-III Changes: A change in manufacturing site to a different campus where same equipment, same environmental conditions and the same controls are used.

Table 9: Test Documentation of Manufacturing site Level-III Changes

\begin{tabular}{|c|c|c|c|}
\hline $\begin{array}{c}\text { Test } \\
\text { documentations }\end{array}$ & IR & SS & MR \\
\hline $\begin{array}{l}\text { Chemistry } \\
\text { documentation }\end{array}$ & \multicolumn{3}{|c|}{ Release notification of change \& updated batch record } \\
\hline $\begin{array}{l}\text { Significant body } \\
\text { of information } \\
\text { available }\end{array}$ & \multicolumn{3}{|c|}{$\begin{array}{l}\text { One batch of three months accelerated study up to three batches } \\
\text { with long term stability study. }\end{array}$} \\
\hline $\begin{array}{l}\text { Significant body } \\
\text { of information is } \\
\text { not available }\end{array}$ & \multicolumn{3}{|c|}{$\begin{array}{l}\text { Up to three batches with three months accelerated study, first } \\
\text { three production batches with long term stability study. }\end{array}$} \\
\hline Dissolution & \begin{tabular}{llr|} 
As per & low \\
permeability & $\&$ & high \\
solubility & & \\
\end{tabular} & Comparison & Extended \& delayed \\
\hline In-vivo & \multicolumn{2}{|l|}{ None } & $\begin{array}{ll}\text { Single } & \text { dose } \\
\text { bioequivalence }\end{array}$ \\
\hline File & \multicolumn{2}{|c|}{$\begin{array}{l}\text { Change being Expected(Accelerated } \\
\text { study ) \& Annual Report(Long term } \\
\text { stability study) }\end{array}$} & $\begin{array}{l}\text { PA (Prior Approval } \\
\text { all data including } \\
\text { accelerated stability } \\
\text { data) AR (Annual } \\
\text { report long term } \\
\text { stability data) }\end{array}$ \\
\hline
\end{tabular}

Batch Size Changes: Batch size changes include change to larger or small production 
batch, less than 100000 units scale down not covered, Scale-up validation needed, may need inspection.

Batch Size Level-I Change: A change up to and including a factor of 10 times pilot/bio- batch where cGMPs, SOPs, controls, formulation, manufacturing procedures etc. are same.

Table 10: Test Documentation of Level-I Batch size Changes

\begin{tabular}{|l|l|l|l|}
\hline \multicolumn{1}{|c|}{$\begin{array}{c}\text { Test } \\
\text { documentations }\end{array}$} & \multicolumn{1}{c|}{ IR } & \multicolumn{1}{c|}{ SS } & \multicolumn{1}{c|}{ MR } \\
\hline $\begin{array}{l}\text { Chemistry } \\
\text { documentation }\end{array}$ & Release notification of changes \& updated batch records \\
\hline Stability study & One long term stability study \\
\hline In vivo & None & Release \\
\hline Dissolution & Release & None \\
\hline File & Annual report (long term stability study) \\
\hline
\end{tabular}

Batch Size Level-II Change: Defined as a SOPs and controls, formulation and change in batch size beyond a factor of 10 times manufacturing procedures are same. the pilot / bio batch where cGMPs, equipment,

Table 11: Test Documentation of Level-II Batch size Changes

\begin{tabular}{|l|l|l|l|}
\hline Test documentation & \multicolumn{1}{|c|}{ IR } & \multicolumn{1}{c|}{ SS } & \multicolumn{1}{c|}{ MR } \\
\hline $\begin{array}{l}\text { Chemistry } \\
\text { documentation }\end{array}$ & Release notification of change \& updated batch records \\
\hline Stability testing & One to three months accelerated study with one long term stability study \\
\hline $\begin{array}{l}\text { Significant body of } \\
\text { documentation }\end{array}$ & $\begin{array}{l}\text { One batch with three months accelerated } \\
\text { stability study \& one long term stability study }\end{array}$ & $\begin{array}{l}\text { Same three batch with } \\
\text { long term stability } \\
\text { study }\end{array}$ \\
\hline Dissolution & $\begin{array}{l}\text { As per low } \\
\text { permeability high } \\
\text { solubility }\end{array}$ & Comparison & Extended \& delayed \\
\hline In vivo & None & \multicolumn{2}{|l|}{} \\
\hline File & $\begin{array}{l}\text { Change being Expected (Accelerated study) \& Annual Report (Long } \\
\text { term stability study) }\end{array}$ \\
\hline
\end{tabular}

Manufacturing Changes: It includes changes that may affect equipment's\& changes that may affect the manufacturing process.

\section{Equipment's Changes:}

Table 12: Test Documentation of Level-I Changes of Equipment changes of Manufacturing changes

\begin{tabular}{|l|l|l|l|}
\hline Test documentation & \multicolumn{1}{|c|}{ IR } & \multicolumn{1}{c|}{ SS } & \multicolumn{1}{c|}{ MR } \\
\hline Chemistry & Release notification of change \& updated batch record \\
\hline Stability testing & One long term & Release \\
\hline Dissolution & Released & None & \\
\hline In vivo & None & \multicolumn{2}{|l}{} \\
\hline File & Annual report long term stability \\
\hline
\end{tabular}


Equipments Changes to Level-II of Manufacturing changes: Changes in equipment to a different design or different operating

Table 13: Test Documentations of Level-II Changes Of Equipments changes of Manufacturing changes

\begin{tabular}{|c|c|c|c|}
\hline Test documentation & IR & SS & MR \\
\hline Chemistry documentations & \multicolumn{3}{|c|}{ Release notifications of changes \& updated batch records } \\
\hline $\begin{array}{l}\text { Significant body of } \\
\text { documentation }\end{array}$ & \multicolumn{2}{|c|}{$\begin{array}{l}\text { One batch with three months accelerated } \\
\text { stability study \& one long term stability } \\
\text { study }\end{array}$} & $\begin{array}{l}\text { Same three } \\
\text { batch with long } \\
\text { term stability } \\
\text { study }\end{array}$ \\
\hline Dissolution & $\begin{array}{l}\text { As per } \quad \text { high } \\
\text { permeability } \& \text { low } \\
\text { solubility }\end{array}$ & Comparison & $\begin{array}{l}\text { Extended \& } \\
\text { Delayed }\end{array}$ \\
\hline In-vivo & \multicolumn{3}{|l|}{ None } \\
\hline File & $\begin{array}{l}\text { PA(Prior Approval } \\
\text { all data including } \\
\text { accelerated stability } \\
\text { data),AR(Annual } \\
\text { report long term } \\
\text { stability data) }\end{array}$ & $\begin{array}{l}\text { CBE(Change } \\
\text { Being Effected) } \\
\text { including } \\
\text { accelerated } \\
\text { stability data, } \\
\text { AR(Annual } \\
\text { report long term } \\
\text { stability data) }\end{array}$ & $\begin{array}{l}\text { PA(Prior } \\
\text { Approval all } \\
\text { data including } \\
\text { accelerated } \\
\text { stability data), } \\
\text { AR(Annual } \\
\text { report long } \\
\text { term stability } \\
\text { data) }\end{array}$ \\
\hline
\end{tabular}

\begin{tabular}{|c|c|}
\hline $\begin{array}{l}\text { Manufacturing } \quad \text { Process } \\
\text { Manufacturing Process Level-I Change: }\end{array}$ & $\begin{array}{l}\text { This category includes changes such as tim } \\
\text { mixing and speeds of operating wi } \\
\text { application and validation ran }\end{array}$ \\
\hline
\end{tabular}

Table 14: Test Documentation of Manufacturing process Level-I changes

\begin{tabular}{|l|l|l|l|}
\hline \multicolumn{1}{|c|}{ Test documentation } & \multicolumn{1}{|c|}{ IR } & \multicolumn{1}{c|}{ SS } & \multicolumn{1}{c|}{ MR } \\
\hline Chemistry documentation & Release & Release & $\begin{array}{l}\text { Release notification of change \& } \\
\text { updated executed batch record }\end{array}$ \\
\hline Dissolution & Release & None & Release \\
\hline In vivo & None \\
\hline File & Annual report \\
\hline
\end{tabular}

Manufacturing Process Level-II Changes: This category includes changes such as time of mixing and speeds of operating outside of application and validation ranges

Table 15: Test Documentation of Manufacturing process Level-II changes

\begin{tabular}{|c|c|c|c|c|}
\hline \multicolumn{2}{|c|}{ Test documentation } & IR & SS & MR \\
\hline \multicolumn{2}{|c|}{ Chemistry documentation } & \multicolumn{3}{|c|}{ Release notification of change \& updated batch records } \\
\hline \multicolumn{2}{|c|}{ Stability testing } & One long term & - & $\begin{array}{l}\text { One batch with three } \\
\text { months accelerated } \\
\text { study \&one long } \\
\text { term stability study. }\end{array}$ \\
\hline stability & $\begin{array}{l}\text { Specific body of } \\
\text { documentations }\end{array}$ & - & $\begin{array}{l}\text { One batch with } 3 \\
\text { months accelerated } \\
\text { stability study. }\end{array}$ & - \\
\hline
\end{tabular}




\begin{tabular}{|l|l|l|l|l|}
\hline & & $\begin{array}{l}\text { First long term } \\
\text { stability study }\end{array}$ & \\
\cline { 2 - 5 } & $\begin{array}{l}\text { Non-specific of } \\
\text { body } \\
\text { documentations }\end{array}$ & $\begin{array}{l}\text { first three production } \\
\text { batch with 3 months } \\
\text { accelerated stability } \\
\text { study }\end{array}$ & - \\
\hline Dissolution & $\begin{array}{l}\text { Low } \\
\text { permeability } \\
\text { high solubility }\end{array}$ & Comparison & Extended \& delayed \\
\hline File & $\begin{array}{l}\text { CBE(Change Being Effected) including accelerated stability } \\
\text { data, AR(Annual report long term stability data }\end{array}$ \\
\hline
\end{tabular}

Manufacturing Process Level-III Changes: such as a change from wet granulation to direct This category includes change in the type of process used in the manufacture of the product, compression of dry powder.

Table 16: Test Documentations of Manufacturing process Level-III changes

\begin{tabular}{|c|c|c|c|c|}
\hline \multicolumn{2}{|c|}{ Test documentations } & IR & SS & MR \\
\hline \multicolumn{2}{|c|}{ Chemistry documentations } & $\begin{array}{l}\text { Release notifications of changes } \\
\& \text { updated batch records }\end{array}$ & \multirow{7}{*}{ 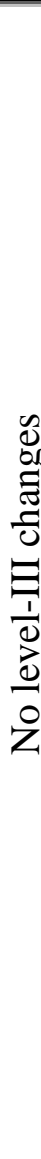 } & $\begin{array}{l}\text { Release notifications of } \\
\text { changes \& updated executed } \\
\text { batch records }\end{array}$ \\
\hline \multicolumn{2}{|c|}{ Stability testing } & & & $\begin{array}{l}\text { Three batches with } 3 \text { months } \\
\text { accelerated stability study, first } \\
\text { three batches with long term } \\
\text { stability study. }\end{array}$ \\
\hline \multirow{2}{*}{ 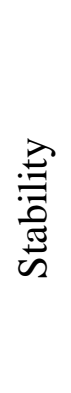 } & $\begin{array}{l}\text { Specific body of } \\
\text { documentations } \\
(\mathrm{SDB})\end{array}$ & $\begin{array}{l}\text { One batch with three months } \\
\text { accelerated study \& one long } \\
\text { term stability study }\end{array}$ & & \\
\hline & $\begin{array}{l}\text { Non-specific body of } \\
\text { documentations } \\
\text { (NSDB) }\end{array}$ & $\begin{array}{l}\text { Up to } 3 \text { batch with } 3 \text { months } \\
\text { accelerated stability study and } \\
\text { up to } 3 \text { batch with long term } \\
\text { stability study }\end{array}$ & & \\
\hline \multicolumn{2}{|c|}{ Dissolution } & $\begin{array}{l}\text { Low permeability high } \\
\text { solubility }\end{array}$ & & Extended \& delayed \\
\hline \multicolumn{2}{|c|}{ In vivo } & Study needed & & Single dose \\
\hline \multicolumn{2}{|c|}{ File } & $\begin{array}{l}\text { PA(Prior Approval all data } \\
\text { including accelerated stability } \\
\text { data), AR(Annual report long } \\
\text { term stability data) }\end{array}$ & & $\begin{array}{l}\text { PA(Prior Approval all data } \\
\text { including accelerated stability } \\
\text { data), AR(Annual report long } \\
\text { term stability data) }\end{array}$ \\
\hline
\end{tabular}

\section{CONCLUSION}

SUPAC-Industry Perspective: it is based on interview with six companies in first half of 1997\& concluded that SUPAC guide line have advantages that:

- Shorter waiting time for site transfers,

- Reducing operating overhead \& maintenance expenses.
- More rapid implementation of process and equipment changes

- Improved yield \& Reduce failure investigations

- More rapid implementation of batch size increases Production of fewer unmarketable stability batches

- Reduce stability testing/costs 
- Reduced administrative costs for documentation of changes by the regulatory affairs departments

- Estimated saving \$51.2 million/year.

\section{ACKNOWLEDGEMENT}

I take this opportunity to express my deep sense of gratitude to all teaching and non-teaching staff of B.K. Mody Govt. Pharmacy College Rajkot, Gujarat, India for his encouragement, guidance and inspiration to write this article.

\section{CONFLICT OF INTEREST}

No conflict of interest is there.

\section{FUNDING SOURCE}

There is no Funding Source. The no any role of study sponsors in the study design, collection, analysis of study and interpretation of data; in the writing of the manuscript and in the decision to submit the manuscript for publication.

\section{REFERENCES}

1. Mendapara VP SN, Purohit PV, Sanghavi G, Ashara KC. SUPAC of Immediate Release Solid Oral Dosage Form-Eplerenone. Inventi Rapid: Pharm Tech. 2013;3:1-7.

2. Azeem S and Sharma S, et al. Immediate release drug delivery systems: a review. Int J Biopharm Toxicol Res. 2011;1(1):24-46.

3. Lawrance MJ. Monographs \& Excipients. In: Convection USP, editor. US 2008.

4. Wiley-Liss inc. The American Pharmacists Association. J Pharm Sci. 2000; 97:1350-60.

5. Carstensen JT, Rhodes CT. Drug Stability: Principles and Practices. 3rd ed; 2006. p. 227-35.

6. Expert Consultation for 2nd Addendum to the 3rd Edn of the Guidelines for Drinking-water Quality. Geneva; 15-19 May 2006.

7. Wiley-Liss inc. The American Pharmacists Association. J Pharm Sci. 2000; 97:1350-60.

8. WHO drug information book, Malta, Vol 12, 3rd ed; 1998.

9. Ashara KC, Paun JS, Soniwala MM, Chavda JR and Badjatiya JK. Nanoparticulate drug delivery system: A novel approach, International Journal of Drug Regulatory Affairs. 2013; 1(2): 39-48.

10. Patel R, Patel KR, Patel NM. Supac guidance for immediate release solid oral dosage forms. Internationale Pharmaceutica Sciencia. 2012; 2(2):35-44. 